\title{
Analyses Of Research For Understanding The Problems of Patients With Diabetes
}

${ }^{1}$ University "Ovidius" of Constanta, Faculty of Medicine

\begin{abstract}
In spite of advances in the management of diabetes mellitus, late complications still represent a problem. Research suggests that improved metabolic control, including the change of lifestyle, which requires the patient's own willingness and ability to adapt, could limit those complications. This article is intended to provide the background to a long-term research programme designed to gain a deeper understanding of diabetic patients' perspectives in their attempt to adapt. It uses the experiences of further groups of diabetic patients, in an attempt to show the usefulness of a cumulative approach towards the building up of a theoretical framework.

Aims of study

This study aims to:

1. give a background to a long-term qualitative theory generating study concerning patients' experiences of managing diabetes.

2. argue that diabetic patients (and nursing as a discipline) might benefit from qualitative research with a focus on the patients' perspective.
\end{abstract}

Keywords: Diabetes, diabetes management, concept 'understanding', selfcare, nursing.

\section{Relu Liviu Craciun}

$3^{\text {rd }}$ Department, Medical Sciences, Faculty of Medicine University "Ovidius" of Constanta

145 Tomis Bvd., Constanta, ROmania, 900591

email : rlcraciun@yahoo.com

phone: +40723296667

\section{Introduction}

It is estimated that 415 million people throughout the world have diabetes mellitus. There are major geographical variations in diabetes in the world, with more than a two-fold increase of incidence in certain areas, pointing out the existence of environmental risk factors.

Since 1922, when insulin was discovered, much energy was necessary to analyse the problems related with diabetes. Discoveries with an enormous impact have been made. No less than three Nobel prizes have been awarded for contributions to diabetes research about insulin [1]. The discovery of insulin ensure a better life and surviving and also the improvement in devices for administration for the treatment increase the acceptability for all patients.

Even more important for the individual patient might be clinical research regarding the treatment of vascular complications. Many patients with proliferative retinopathy have probably kept their visual ability due to better collaboration with the eye specialist. The possibilities for treating even lifethreatening conditions, like uremia due to diabetic nephropathy, with dialysis and renal transplantation, have meant an improvement in the fate of those patients.

However, the self-management of diabetes 
requires the patient's own willingness and ability to adapt, which is an area for inquiry that has taken a backseat to more glamorous and instantly gratifying forms of high-tech research. Better control of glycemia, blood pressure and weight will prevent the chronic complication related to diabetes and all this are beneficial for day to day life.

\section{Theoretical Perspective}

The clinical studies, to which this paper provides a background, is based on an interactional perspective, which is an obligatory condition to find how people interpret their real life. It attempts to increase the understanding of human behavior from the patient own frame of reference.

The concept 'understanding' is important if we want to choose the particular way both for the datacollection and the analysis. Underlying the study is the assumption that learning to manage any chronic condition like diabetes is a difficult and long process, which involve a collaboration between conscious and unconscious resources that doctors, patients and nurses have. The model for the research approach in this study is grounded theory [2] which is based on a social psychological perspective, named symbolic interactionism. Elizabeth K. Babler [3] summarizes the concept of symbolic interaction thus:

- The first premise is that patients and their families act different in relation with their understanding. This means that they have their own perceptions about important things in life

- The second premise is that the importance of different things are evaluated by the social interaction that one has about his problem or disease.

- The third premise is that.

The concern of this study is how diabetic patients control their diabetes and the comorbidities that they have. The individual is active in making decisions, not only by on his experience, but also by the knowledge related to the disease and by the experience of people around him [4], where past experiences is used for expectations about the futures and expectations.

Besides the concerns that they have, the study analyzed what are others factors could interfere with their medical condition that can improve or, on the contrary, can degrade their life. The most important things for a better metabolic control for patients with diabetes is better understanding of all information they received, importance of new life style, selfmonitoring and control of all.

A better understanding of patients and medical staff is essential in improving the evolution of diabetes and a good point is to use diabetic patients themselves as informants.

\section{Data From Literature}

Data from clinical studies shows that there are three important coordinates in diabetes care:

(a) care of patient by the medical team: doctor and dietician

(b) nursing for patient with complications diabetes

(c) qualitative approach in patients with

Care of patient by the medical team: doctor and dietician

Treating diabetes to obtain glycemic target or at least improve metabolic control is the best way to optimize the life style. The message that we have to deliver to diabetic patients is that life style changes and treatment are born stone for preventing complications and have an active normal life. The patient and the family has be informed about:

- Importance of adhering to the treatment and diet

- Having an active life as this can improve the metabolic control because of physical effort and psychological environment

- Taking the medication

Explaining that the disease play an important 
part in life could be of great help in integration of persons with diabetes in social life [5].

The time of diagnosis and moment of introduction of insulin therapy have a very important psychological impact to be managed with great care. Most often in the watershed moments of the disease occur concerns that can lead to decreased compliance to diet and treatment with serious repercussions on further developments. "Stories" circulating around insulin therapy considerably reduces the acceptability of treatment. Therefore prior discussion with the patient in office is very important. The discussion in the educational room with more patients having the same problem is extremely useful and help understand the mechanisms that determine the need for this therapy [6].

Vascular complications is another milestone because in these situations is more difficult management disease and metabolic control should be effective to prevent situations where the patient's life is endangered [7,8]. In 1980 the life expectancy of children aged 10 years with diabetes was about 55 years [4]. Today, given advances in medical treatment, things have changed and increased lifetime equaling people without diabetes. From this point of view new therapies and extensive use of insulin analogs has made life easier. In this context self-monitoring patients at home, has made it possible to manage the disease by the physician in conjunction with the patient.

Considering the fact that metabolic control is a means of achieving a normal life and a better quality of life, the care of diabetic patients is in general difficult [1]. In the recent literature there is a concern about the paradox, 'that we believe that we know so much about diabetes but still are not able to reach the goal to limit the late complications' [1].

Luft identified three important things for good control of patients with diabetes:

1. Relation between our knowledge about diabetes and theoretical perspectives

2. Practical approach to each patient taking into account the particularities compared with known diabetic population

3. Relation of diabetes as a chronic disease and social and psychological implications.

Nursing for patient with complications
The patient is the most important person along with the doctor in the management of diabetes. For patient diagnosed as having diabetes, is not necessarily to define as having a chronic disease, but we have to care also about his own experience with his disease [1]. Non-compliant patients are those who have concerns about the control of their disease [9]. This type of patients need important support and more attention as they need a lot of information and psychological support. Non-compliant behavior can be induced by complicated circumstances.

People act differently depending on their interpretations of the general situation.

Diabetic patients and their families need help and support from the health care system, in order to control the complex relations between nutrition, insulin treatment, life style changes and emotions. It has already been implied that diabetic patients, all the patients with chronic conditions, need more 'caring' than 'curing.

Caring means management with patients rather than management of diseases.

\section{diabetes. \\ Qualitative approach in patients with \\ Research that intend to link medicine's} definition of the disease with the particular issues of the patient's situation has to come from a qualitative approach in patients with diabetes. There are various qualitative approaches such as anthropology, ethno science that can all be used as modes of important things concerned with understanding people and their actions from their level of understanding. It is not possible to explain phenomena in the social world in a scientific quantitative manner. Concepts like understanding, expectations, desires, prediction about future are involved in the social world and require different modes of explanation [10]. People act different on the basis of their own interpretation and knowledge of the meaning that they have in different modes of behavior.

One interpretation of a paradigm is 'the entire constellation of beliefs, values, techniques, and so on shared by the members of a given community' [11]. 


\section{Data Analysis And Methodology}

\section{Method of study}

In this study, we used the interview method. Thus, we selected patients with diabetes, in various stages of disease progression and different therapeutic approach, as a conversation, individual or family, on patient desire and we kept in touch by phone when the patient felt the need communicate or had various questions to the medical staff. We try to render correct information provided by the patient so that the contribution of doctors or nurses to be as small as not to influence the results. We encouraged the patient to disclose all fears of disease, treatment or have repercussions on the future and family involved to create an atmosphere of relaxation.

We included 123 patients in the study, of which remained only 78 completed the study.

Those who refused to continue the study cited in the majority lack of time and fear of discussions to find out information about possible complications of diabetes [11].

\section{Selection of respondents}

Choosing the 78 respondents included three groups of patients with insulin-dependent, with different experiences in terms of disease, presence of complications and insulin schemes. This choice was made so as to cover several disease models approach.

Getting groups with different approaches increases the likelihood that the physician that collect data can get different results in order to fit patients in class that compare them later to addressing right between groups in the same group [4]. Therefore we chose the comparative analyzes that have been made within groups and between groups.

- The first group included 21 insulin-dependent diabetic patients, selected by their physician as being well adapted and with no known vascular complications, in spite of long duration of diabetes. Data from the first 20 interviews from this group were analyzed separately in the light of the respondents' effective self-management. Five conceptual categories, as an account of these respondents' experiences in dealing with diabetes, emerged.

1. Patient can manage the disease by himself, is his own responsibility and has tools to control this situation.

2. Walking is a good way to control the disease, but is afraid that will not go sufficiently and thus cannot meet its obligations related to everyday life.

3. Life expectancy is low and eventually will die much earlier than the situation that did not have diabetes.

4. It is not sick, but having a chronic condition cannot assume the sick role, though he thinks it might have complications related to diabetes which cannot manage.

5. Being dependent self-medication can control very well the disease, but carefully plan the actions.

The five categories can be seen as a category for the presentation of the issues raised by these first selected participants. However, a substantive theory on the process of managing diabetes means elaborating and linking categories to some kind of pattern. Hence, in order to go further with the theory generating process, it was necessary to involve respondents in the study who might have additional or contradictory experiences.

- 27 respondents with complications of diabetes were selected as the second comparison group, and the categories mentioned above were used as a hidden agenda for the first interviews with this group. It was thought that respondents in this phase of the career of being a diabetic patient could contribute even more experiences on what it means to have diabetes, with all its consequences. They might thus contribute to the extension of empirical knowledge.

- 30 newly diagnosed diabetic patients were selected as the third group. They were selected to repeated interviews to see how they had learned to manage their situation, from a practical and psychological point of view. 


\section{Data Collection And Analysis}

In order to achieve this study were conducted informal interviews as a conversation. It was used an agenda that allowed the interviews to be conducted in the same manner for all participants and contained the same type of questions. All data were collected by the author. Once respondents were informed that there was an interest in learning from them about their current situation, they were asked to introduce topics that they considered to be relevant. Initial project was designed to include only theoretical knowledge of the subject they learned from discussions with medical staff.

Although concentrated, interviews were conducted in such a way that the influence of the man who led the interview to be as small. Benchmarking method made it possible to concentrate progressively questions so as to make them as clear and can be processed $[12,13]$. When interviews were repeated with the same person (as in most cases), data from earlier occasions were carefully examined before subsequent interviews. In this way, questions were deepened and interviews became more purposeful.

It is the underlying structure which distinguishes a purposeful interview from a social conversation. There is a fine balance basing an interview on a serious research and theoretical possibilities, while still allowing the respondent to introduce new thoughts and concepts [14]. Special attention was given during the interview adoption from the beginning of the concepts that give a certain direction theoretical interview.

In contrast to the structured interview, no standardization of data has not been made in advance

\section{Health Policy Implications}

Despite encouraging advances in medical management of diabetes, long-term complications could not be prevented. For patients with diabetes need to find a strong motivation to stimulate effective disease control, their own desire and involvement in management of diabetes. That point is discussed in advance with the patient so as to identify human factors that cause an increased adherence and motivation necessary to understand the complexity of medical interventions [15]. Therefore the patient himself must learn to understand his own will and motivation, and his illness.

Only a holistic model of care based on informed participation of patients has succeeded. The conclusion is that diabetes care policy should be based on individualized care models. The information provided by patients with diabetes itself must be included in the new models of care. Policy that ignores this information will not be effective in diabetes patient care and fails to prevent chronic debilitating complications that represent a burden for the patient and for society.

\section{CONGLUSIONS}

The conclusions are derived from two types of approach. A theoretical approach that refers to literature that deals with this issue, but from this point of view there are few publications that have examined this issue. Practical approach refers to patients with diabetes itself, the experience of talking to doctors and nurses. The literature has shown that there are gaps in knowledge on care and communication with patients with diabetes as opposed to treatment of patients with diabetes which is complex and does not account for understanding the patient if he is properly informed.

Another conclusion that emerges from all the studies that had this issue is the failure to prevent long-term complications arise because of the rigidity of medical system that is based more on therapy and not on individual patient needs.

Interviews with patients with diabetes have highlighted their difficulties, fears and anxieties, partly due to their lack of understanding of the 
disease or because the approach in the same way to all patients with stereotypes that would be eliminated.

Rich information provided by these studies show the need and their desire for a wider concern of the health system to include in their programs of care problems in the family, the workplace and society in general.

This paper identifies general problems of diabetes patient care, but can not provide a definitive model for care in the future, his message is that each individual patient must be addressed, and that treating all patients in the same manner we will not get the results that we are waiting for.

\section{References}

1. Luft R. (1986). Filling the gaps. Presentation at WHO Conference on Diabetes Care as a Model for Primary Health Care. Karolinska Institute, Stockholm.

2. Chenitz, W. C., \& Swanson, J. M. (1986). Qualitative research using grounded theory. In W. C. Chenitz \& J. M. Swanson (Eds.), From practice to grounded theory (pp. 3-15). Menlo Park, CA: Addison-Wesley.

3. Babler, E.K. \& Strickland, C.J. (2000). Moving the journey towards independence: Adolescents transitioning to successful diabetes selfmanagement P17-19.

4. Preventing insulin dependent diabetes mellitus: the environmental challenge. Diabetes Epidemiology Research International. (1987). $\mathrm{Br}$ Med J (Clin Res Ed). 295(6596), 479-481.

5. Denzin, N.K. \& Lincoln, Y.S. (2000). The SAGE Handbook of Qualitative Research: Sage Publications.

6. Sackett, D.L., Straus, S.E., Richardson, W.S., Rosenberg, W. \& Haynes, R.B. (2000). Evidence Based Medicine: How to Practice and Teach $E B M$. London: Churchill Livingstone

7. Morse, J.M., Penrod, J. \& Hupcey, J.E. (2000). Qualitative outcome analysis: evaluating nursing interventions for complex clinical phenomena. $J$ Nurs Scholarsh. 32(2), 125-130..

8. Miller,W.R.(2010).QualitativeResearchFindings as Evidence: Utility in Nursing Practice. Clinical Nurse Specialist CNS, 24(4), 191-193. http://doi. org/10.1097/NUR.0b013e3181e36087.

9. Barbour R. (2000). The role of qualitative research in broadening the "evidence base" for clinical practice. J Eval Clin Prac. 6(2), 155163.

10. Cohen, D.J. \& Crabtree, B.F. (2008). Evaluative criteria for qualitative research in health care: controversies and recommendations. Ann Fam Med. 6(4), 331-339. doi: 10.1370/afm.818

11. Baker, G.R. (2011). The contribution of case study research to knowledge of how to improve quality of care. BMJ Qual Saf. 20 Suppl 1, i3035. doi: 10.1136/bmjqs.2010.046490.

12. Dougherty, D. \& Conway, P.H. (2008). The „3T's” road map to transform US health care: the "how” of high-quality care. JAMA. 299(19), 2319-2321. doi: 10.1001/jama.299.19.2319

13. Berwick, D.M. (2008). The science of improvement. JAMA. 299(10), 1182-1184. doi: 10.1001/jama.299.10.1182

14. Sparkes, A.C. (2001). Myth 94: qualitative health researchers will agree about validity. Qual Health Res. 11(4), 538-552. doi: 10.1177/104973230101100409

15. Speziale, H.S. \& Carpenter, D.R. (2007). Qualitative Research in Nursing: Advancing the Humanistic Imperative: Lippincott Williams \& Wilkins. 